\title{
A tracing method for the botanical origins of six geographical indication (GI) jujube species by the crude polysaccharides characterization
}

Kai Mao ( $\square$ maokai@sxau.edu.cn )
Shanxi Agricultural University

\section{Chun Yang}

Shanxi Agricultural University

\section{Weiying Ding}

Shanxi Agricultural University

Jiangning Zhang

Shanxi Agricultural University

Zheng Ye

Shanxi Agricultural University

Jiming Han

Shanxi Agricultural University

\section{Ling Zhang}

Shanxi Agricultural University

\section{Research Article}

Keywords: Geographical indication (GI), Botanical origins, Jujube species, Crude polysaccharide, Molecular weight (Mw) distribution, Monosaccharide composition, Functional groups and bonds

Posted Date: March 3rd, 2022

DOI: https://doi.org/10.21203/rs.3.rs-1402556/v1

License: (1) (i) This work is licensed under a Creative Commons Attribution 4.0 International License. Read Full License 


\section{Abstract}

\section{Background}

Current official standards of geographical indication (GI) jujube species only employed simple parameters like appearance, grown region as distinguishing indicators, but they could not tell the subtle differences. In addition to the genetic method, the most efficient and accurate way to trace botanical origins at present, some researchers successfully tracing the geographical or botanical origin by the content or characteristics of biomacromolecule or functional ingredients such as metabolomics and polysaccharide. This article was to develop an efficient way to trace the botanical origins of $6 \mathrm{Gl}$ jujube species by crude polysaccharide characterization.

Results

The crude polysaccharides from 6 geographical indication (GI) jujube species of Shanxi province were tested with the parameters of purity, uronic acid content, $M_{w}$ distribution, monosaccharide composition, functional groups and bonds. In the purity and uronic acid content assay, the purity varied within a very narrow band (96\%-99\%) and presented no negative or positive correlation with uronic acid content. In the experiment of $M_{w}$ distribution by HPGPC, five peaks (RT1, RT2, RT3, RT4, RT5) were almost observed in 6 jujube species, and the content of RT3 (240-250 Da), with significant correlation to the content of RT4 and RT5, exhibited significant differences among 6 jujube species (RSD $=1.28>1.00)$. The monosaccharides composition indicated that the polysaccharides from 6 jujube species were rich in glucose (55-75\%), arabinose (10-15\%) and galactose acid (10-20\%), with small amounts of rhamonose, glucosamine hydrochloride, galactose, xylose, mannose (less than $5 \%$ ). The FT-IR spectrum showed that crude polysaccharides from six jujube species shared similar functional groups and chemical bonds.

\section{Conclusions}

The results above indicated that the $M_{w}$ distribution of crude polysaccharides showed both homogeneity and differences in the $6 \mathrm{Gl}$ jujube species. First of all, five peaks (RT1, RT2, RT3, RT4, RT5), respectively representing the $\mathrm{M}_{\mathrm{w}}$ of 186646-285262 Da (RT1), 4634-17296 Da (RT2), 240-250 Da (RT3), 98-103 Da (RT4) and 57-64 Da (RT5), could be detected in all 6 jujube species. Secondly, the RT3 contents exhibited significant differences among 6 jujube species $(R S D=1.28>1.00)$ and showed significant correlation with RT4 and RT5. Therefore, the $\mathrm{M}_{\mathrm{w}}$ distribution may be treated as a potential distinguishing indicator among 6 jujube species.

\section{Background}

Jujube or Chinese jujube (Zizyphus jujuba Mill.) is a traditional Chinese herb widely distributed in Asia, Europe and Australia, and especially in northern China[1]. As illustrated by animal and cell experiments, positive bio-activities were found in different organs, including leaves, flowers and fruits[2]. 
Comparatively, the jujube fruit, known as Chinese date or red date, contains various bioactive substances such as vitamin C[3], phenolics[4], flavonoids[5], triterpenic acids[6, 7], and polysaccharides, possesses considerably potential nutrition value. Due to its considerable nutritional and nutraceutical values, the jujube fruit is also treated as a traditional Chinese medicine (TCM) for the remedy of anorexia, bloodnourishing, fatigue and so on[8].

Previous studies revealed more than 700 cultivars have been found in china, and Shanxi province, one of the jujube origins in northern China, has particularly contributed to the largest amounts of geographical indications (GI) Chinese jujube species[9]. Among them, Jishanbanzao, Jiaochengjunzao, Pinglutuntunzao, Taiguhupingzao, Baodeyouzao and Linxianmuzao were the most famous 6 Gl jujube species with the largest export volume. But current Chinese official standards of the the $6 \mathrm{GI}$ jujube species could not tell the subtle differences, because they simply employed appearance characteristics and grown regions as the specific indicator.

Absolutely, genetic identification method is the most precise and efficient method and it has been proved that 622 unique genotypes can be selected from 962 jujube species by 24 SSR markers[10]. However, the contents or characteristics of biomacromolecule or functional ingredients had also been successfully used as indicators for tracing the botanical origins. For example, molecular fingerprint (the content of citrate, lysine and 10-hydroxy-2-decenoic acid) analysis by nuclear magnetic resonance (NMR) spectroscopy could be treated as the identification indicator to successfully certify and trace the botanical and geographical origin of sophora pollen residues in royal jelly[11]. Besides, the $M_{w}$ (molecular weight) distribution and monosaccharide composition analysis of polysaccharides could be employed as the tracing indicators to classified the 4 species of Polygonatum spp. into 2 different groups reflecting the two different botanical origins.

As for us, crude polysaccharide characterization could be the potential way to trace the botanical origin of jujube species. Polysaccharides are the most important constituents in fresh jujube (20\% w/w)[12], and are featured by complex structures, wide range of $\mathrm{M}_{\mathrm{w}}$ distribution.

In order to trace the significant indicators as much as possible, we utilized HPGPC (high-performance gel permeation chromatography) method which is more sensitive than HPLC (high performance liquid chromatography) method for profiling $\mathrm{M}_{\mathrm{w}}$ distribution. And IC (ion chromatography) method was used to measure monosaccharide composition with 16 monosaccharides standards (more than that in most papers because 5 monosaccharides standards were purified by our own lab).

To our knowledge, there have been no researches on tracing the botanical origins of jujube species by the polysaccharides $\mathrm{M}_{\mathrm{w}}$ distribution, structure, monosaccharide composition and so on. Hence we picked out 6 representative GI jujube species of Shanxi province, measured the crude polysaccharides contents and characteristics, and developed an efficient tracing indicators with significant difference.

\section{Materials And Methods}




\section{Materials}

Six GI Chinese jujube species of Shanxi province employed in our study were all collected from the origin regions (Table 1 and Fig. 1). They were harvested at red and ripened stage in April.

Table 1 Brief introduction of six GI jujube species from Shanxi province

\begin{tabular}{|llll|}
\hline Popular name & Species & Codes & Geographical origin \\
\hline Jishanbanzao & Ziziphus Jujuba cv. Banzao & ZB & Jishan county, Shnaxi proince \\
\hline Jiaochengjunzao & Ziziphus Jujuba cv. Junzao & ZJ & Jiaocheng county, Shnaxi proince \\
\hline Pinglutuntunzao & Ziziphus Jujuba cv. Tuntunzao & ZT & Pinglu county, Shnaxi proince \\
\hline Taiguhupingzao & Ziziphus Jujuba cv. Hupingzao & ZH & Taigu county, Shnaxi proince \\
\hline Baodeyouzao & Ziziphus Jujuba cv. Youzao & ZY & Baode county, Shnaxi proince \\
\hline Linxianmuzao & Ziziphus Jujuba cv. Muzao & ZM & Lin county, Shnaxi proince \\
\hline
\end{tabular}

\section{Crude polysaccharides preparation}

The fresh fruits were cleaned, sliced and air-dried at $70^{\circ} \mathrm{C}$. The dried fruit $(50 \mathrm{~g})$ bodies were pulverized into powder and extract twice with distilled water $(1: 10 \mathrm{w} / \mathrm{v})$ at $90^{\circ} \mathrm{C}$ (3 h each time). Subsequently, supernatant was obtained by filtration and concentrated to one-tenth of the original volume at $65^{\circ} \mathrm{C}$ by rotary evaporation[13]. The concentrated solution was deproteinated by Sevag method[14] and precipitated with four volumes of ethanol at $4^{\circ} \mathrm{C}$ for $24 \mathrm{~h}$. Then the crude polysaccharides were obtained by precipitate collection with centrifugation, dissolution in distilled water, and lyophilization.

The crude polysaccharides of six jujube species (ZBP, ZJP, ZTP, ZHP, ZYP, ZMP) were all extracted with the methods above.

\section{Total sugar and uronic acid content}

Total sugar content were determined by the phenol-sulphuric acid method[15]. However, the phenolsulfuric acid reagent can also react with uronic acid (or toluene derivatives), which interfering with the accurate determination of polysaccharides purity[16]. Therefore the uronic acid content was Individually determined by the hydroxybiphenyl method[17].

\section{Molecular weight $\left(\mathrm{M}_{\mathrm{w}}\right)$ distribution}

The prepared 6 crude polysaccharides (ZBP, ZJP, ZTP, ZHP, ZYP, ZMP) were analyzed by high-performance gel permeation chromatography (HPGPC) on a Shimadzu LC-10A system equipped with a BRT104-102 column $(8 \mathrm{~mm} \times 300 \mathrm{~mm})$ and a refractive index detector $(\mathrm{Rl}-10 \mathrm{~A})$. The mobile phase $(0.05 \mathrm{M}$ sodium sulfate solution) was set at a constant rate of $0.6 \mathrm{~mL} / \mathrm{min}, 40^{\circ} \mathrm{C}$. The samples were prepared as $5 \mathrm{mg} / \mathrm{L}$ 
solution and centrifuged at $12000 \mathrm{rpm}$ for $10 \mathrm{~min}$. The supernatant was filtered by $0.22 \mu \mathrm{m}$ membrane then transferred to $1.8 \mathrm{~mL}$ val. The standard curve of molecular weight was prepared by dextran standards ( $M_{w} 1152,5000,11600,23800,48600,80900,148000,273000,409800,667800$ Da) (Sigma). According to $\operatorname{lgM}_{\mathrm{w}}-\mathrm{RT}$ calibration curve of dextran standards, the samples molecular weight were determined.

\section{Monosaccharide composition}

Monosaccharide composition was measured by ion chromatograph (IC) with the 16 standard monosaccharides including mannose (Man), manuronic acid (Man A), rhamnose (Rha), galacturonic acid (Gal A), galactose (Gal), glucose (Glc), glucuronic acid (Glc A), arabinose (Ara), xylose (Xyl), fucose (Fuc), glucosamine hydrochloride, N-acetyl-D-glucosamine, D-frutose (D-Fru), D-ribose (D-Rib), Galactosamine hygrochloride, guluronic acid. According to Wang[18], $10 \mathrm{mg}$ crude polysaccharides (ZBP, ZJP, ZTP, ZHP, ZYP, ZMP) were placed in an ampoule, added with $10 \mathrm{~mL}$ TFA (3 M), then hydrolysed for $3 \mathrm{~h}$ at $120^{\circ} \mathrm{C}$. The acid hydrolysate were transferred into a tube, dried with $\mathrm{N}_{2}$, and mixed with $10 \mathrm{~mL}$ of distilled water. Next $100 \mu \mathrm{L}$ solution was sucked into $900 \mu \mathrm{L}$ deionized water and centrifuged at $12000 \mathrm{rpm}$ for $5 \mathrm{~min}$. The supernatant was analyzed by ICS5000 ion chromatograph (Thermo Fisher) equipped with an electrochemical detector. The chromatographic conditions were set as follows: column, DionexCarbopac ${ }^{\text {TMPA2 }}$ (3*150); mobile phase, A: $\mathrm{H}_{2} \mathrm{O}, \mathrm{B}: 15 \mathrm{M} \mathrm{NaOH}, \mathrm{C}: 15 \mathrm{M} \mathrm{NaOH}$ and $100 \mathrm{mM} \mathrm{NaOAc}$; follow rat, $0.3 \mathrm{~mL} / \mathrm{min}$; column temperature, $30^{\circ} \mathrm{C}$.

\section{Fourier-transform infrared (FT-IR) spectrum}

The 6 samples (ZBP, ZJP, ZTP, ZHP, ZYP, ZMP) $(2 \mathrm{mg})$ were pressed into $1 \mathrm{~mm}$ pellets with $\mathrm{KBr}$ powder $(200 \mathrm{mg}$ ). Then they were analyzed by FT-IR650 spectrophotometer (Tianjing Gangdong Sci. \&Tech. Co., Ltd.) in the vibration region of $4000-500 \mathrm{~cm}^{-1}$.

\section{Results And Discussion}

\section{Total sugar and uronic acid contents analysis}

The samples were processed according to 2.2, and glucose and galacturonic acide were used as standards for determination of total sugar and uronic acid content. As the result, total sugar content of the crude polysaccharides (ZBP, ZJP, ZTP, ZHP, ZYP, ZMP) were at range of $96 \%-99 \%$, and $10 \%-23 \%$ uronic acid were found in each sample (Table 2).

Table 2 The purity and uronic acid content of crude polysaccharides in each sample 


\begin{tabular}{|lll|}
\hline Codes & Total sugar content (Purity) \% & Uronic acid content \% \\
\hline ZBP & 97.5 & 11.9 \\
\hline ZJP & 96.9 & 22.1 \\
\hline ZTP & 96.1 & 15.0 \\
\hline ZHP & 98.0 & 16.4 \\
\hline ZYP & 98.0 & 19.5 \\
\hline ZMP & 99.0 & 22.1 \\
\hline
\end{tabular}

The states above showed that the purity varied within a very narrow band and presented no negative or positive correlation with uronic acid content. So the purity and uronic acid contents might not be the suitable tracing indicator at all.

\section{Molecular weight $\left(\mathrm{M}_{\mathrm{w}}\right)$ distribution analysis}

Generally speaking, the molecular weight $\left(\mathrm{M}_{\mathrm{w}}\right)$ distribution is an important bio-chemical parameter to characterize the homogeneity of species[19]. For the detection means, HPLC is generally used in $M_{w}$ measurement. But in our study, the samples were treated as the description in 2.2 and $\mathrm{M}_{\mathrm{w}}$ distribution were measured by HPGPC-RID method which is more sensitive than HPLC method for profiling $M_{w}$ distribution.

Table $3 \mathrm{M}_{\mathrm{w}}$ distribution by HPGPC method and the RSD analysis

\begin{tabular}{|llllllll|}
\hline \multirow{2}{*}{ RT(min) } & \multicolumn{7}{l}{ Peak area ratio/contents $\%$} \\
& ZBP & ZJP & ZTP & ZHP & ZYP & ZMP & RSD \\
\hline RT1 (34.7-35.6) & 11.488 & 33.453 & 16.458 & 23.235 & 36.930 & 26.647 & 0.39 \\
\hline RT2 (41.0-44.0) & 2.743 & 2.161 & 2.079 & 3.378 & 2.824 & 2.183 & 0.20 \\
\hline RT3 (50.6-50.7) & 4.913 & 35.709 & 3.772 & 25.393 & 0 & 0.809 & 1.28 \\
\hline RT4 (52.6-52.8) & 28.244 & 9.225 & 26.672 & 15.411 & 19.389 & 23.617 & 0.35 \\
\hline RT5 (53.9-54.0) & 52.613 & 19.453 & 51.019 & 32.583 & 40.857 & 46.744 & 0.31 \\
\hline
\end{tabular}

Note: For example, RT1 (34.7-35.6): retention time 1 (the peak time ranged from 34.7 to $35.6 \mathrm{~min}$ ); RSD: relative standard deviation.

Table 4 The correlation analysis on polysaccharide contents of different $\mathrm{M}_{\mathrm{w}}$ 


\begin{tabular}{|c|c|c|c|c|c|c|}
\hline & & RT1 & RT2 & RT3 & RT4 & RT5 \\
\hline \multirow[t]{2}{*}{ RT1 } & Pearson correlation & 1 & -0.026 & 0.247 & -0.714 & -0.653 \\
\hline & Significance & & 0.961 & 0.637 & 0.111 & 0.160 \\
\hline \multirow[t]{2}{*}{ RT2 } & Pearson correlation & & 1 & 0.136 & -0.145 & -0.098 \\
\hline & Significance & & & 0.798 & 0.784 & 0.853 \\
\hline \multirow[t]{2}{*}{ RT3 } & Pearson correlation & & & 1 & $-0.851^{*}$ & $-0.895^{\star}$ \\
\hline & Significance & & & & 0.032 & 0.016 \\
\hline \multirow[t]{2}{*}{ RT4 } & Pearson correlation & & & & 1 & $0.993^{\star \star}$ \\
\hline & Significance & & & & & 0.000 \\
\hline \multirow[t]{2}{*}{ RT5 } & Pearson correlation & & & & & 1 \\
\hline & Significance & & & & & \\
\hline
\end{tabular}

Note: ${ }^{*} \mathrm{P}<0.05$ significant, ${ }^{\star \star} \mathrm{P}<0.01$ highly significant.

As shown in Fig. 2 and details in table 3, five peaks represented different $M_{w}$ at the range of 186646285262 Da (RT1), 4634-17296 Da (RT2), 240-250 Da (RT3), 98-103 Da (RT4) and 57-64 Da (RT5).

Obviously, the $M_{w}$ of RT3, RT4 and RT5 belonged to the $M_{w}$ of oligosaccharides or monosaccharides, but they still could be used as the tracing indicator if their distributions showed significant differences.

In HPGPC profiles, five peaks (RT1, RT2, RT3, RT4, RT5) were almost observed in 6 jujube species (only the peak of RT3 was absent in ZYP) and this might be due to the close relationship of 6 jujube species.

According to the RSD and correlation analysis (table 3 and 4), RT3 contents exhibited significant differences among 6 jujube species (RSD $=1.28>1.00)$ and showed significant correlation with RT 4 and RT5. RT3 contents was negatively correlated with RT 4 and RT 5 contents, while RT4 and RT 5 showed a highly significant positive correlation.

Taken together, $M_{w}$ distribution of 6 jujube species not only presented the homogeneity each other but also exhibited significant differences among 6 jujube species. Therefore, $M_{w}$ might prove to be a potential tracing indicator among the 6 jujube species.

\section{Monosaccharide composition analysis}

Monosaccharide composition can be measured by the methods of HPLC-RID (high performance liquid chromatography), GC-MS (gas chromatography mass spectrometry) and IC (ion chromatography). Previous study showed that the uronic acid may not be detected with normal alkaline mobile phase by HPLC-RID method[20], and to our knowledge, up to 7 monosaccharides can be detected by GC-MS method[21]. 
At present, ion chromatography is an essential method for the determination of monosaccharides with the most types, without reducing uronic acid, simple sample treatment and high resolution. Normally more than 13 monosaccharide can be detected[18], and the uronic acid content can be determined without special derivatization reaction. Therefore, the ion chromatography was used to determine monosaccharide composition in this article.

Table 5 The contents of different monosaccharides in ZBP, ZJP, ZTP, ZHP, ZYP and ZMP

\begin{tabular}{|c|c|c|c|c|c|c|c|}
\hline Monosaccharide content \% & ZBP & ZJP & ZTP & ZHP & ZYP & ZMP & RSD \\
\hline \multicolumn{8}{|l|}{ Fuc } \\
\hline \multicolumn{8}{|l|}{ GalN } \\
\hline Rha & 2.5 & 2.5 & 1.8 & 1.9 & 2.5 & 1.9 & 16.0 \\
\hline Ara & 7.1 & 12.6 & 9.5 & 9.9 & 14.9 & 12.5 & 25.0 \\
\hline GlcN & 0.1 & 0.4 & 0.3 & 0.4 & 0.4 & 0.3 & 37.0 \\
\hline Gal & 3.0 & 3.9 & 2.3 & 3.5 & 4.4 & 3.2 & 22.0 \\
\hline Glc & 75.7 & 56.1 & 70.4 & 64.8 & 60.1 & 62.4 & 11.0 \\
\hline \multicolumn{8}{|l|}{ GlcNAc } \\
\hline Xyl & 0.8 & 1.5 & 1.0 & 1.1 & 0.6 & 0.5 & 40.0 \\
\hline Man & 0.7 & 1.0 & 0.8 & 1.0 & 1.1 & 0.8 & 17.0 \\
\hline \multicolumn{8}{|l|}{ Fru } \\
\hline \multicolumn{8}{|l|}{ Rib } \\
\hline GalA & 10.1 & 20.1 & 14.0 & 17.4 & 16.0 & 18.4 & 22.0 \\
\hline \multicolumn{8}{|l|}{ GulA } \\
\hline \multicolumn{8}{|l|}{ GlcA } \\
\hline ManA & & & & & & & \\
\hline
\end{tabular}

As shown in Fig. 3 and table 5, the same monosaccharide composition (Rha, Ara, GlcN, Gal, Glc, Xyl, Man, GaIA) were detected in all polysaccharides by ion chromatography method. The polysaccharides from 6 jujube species were rich in glucose between $55 \%-75 \%$, arabinose and galactose acid contents were almost at range of $10 \%-20 \%$, while the other 5 monosaccharides were less than $5 \%$.

Generally speaking, a relative standard deviation (RSD) greater than 1.0 indicates that most of the values in a set of data are significantly different from their mean. Therefore, the RSD of each monosaccharide content all less than 0.5 (table 4) indicating that the monosaccharide composition could not distinguish the 6 jujube species. 


\section{FT-IR spectrum analysis}

The functional groups of jujube polysaccharide can be characterised by FT-IR spectrum[22, 23]. As shown in Fig. 4 and table 6, the functional groups and chemical bonds of crude polysaccharides from 6 jujube species seemed roughly similar. All the samples showed stretching viberations of $-\mathrm{OH}$ at around (3600$3200 \mathrm{~cm}^{-1}$ ), which located in typical absorption band of polysaccharides.

Table 6 Functional groups and chemical bonds of polysaccharides from 6 jujube species

\begin{tabular}{|c|c|c|c|c|c|c|}
\hline Functional groups or chemical bonds by IR & ZBP & ZJP & ZTP & ZHP & ZYP & ZMP \\
\hline $\begin{array}{l}\text { typical absorption peaks of polysaccharides (3200- } \\
3600 \mathrm{~cm}^{-1} \text { ) }\end{array}$ & $\sqrt{ }$ & $\sqrt{ }$ & $\sqrt{ }$ & $\sqrt{ }$ & $\sqrt{ }$ & $\sqrt{ }$ \\
\hline Existence of sugar ring $\left(2920-2935 \mathrm{~cm}^{-1}\right)$ & $\sqrt{ }$ & $\sqrt{ }$ & $\sqrt{ }$ & $\sqrt{ }$ & $\sqrt{ }$ & $\sqrt{ }$ \\
\hline $\begin{array}{l}\text { existence of uronic acid }(\mathrm{C}=\mathrm{O} \text { stretching viberation and } \\
\mathrm{O}-\mathrm{H} \text { bending vibration) }\left(1500-1740 \mathrm{~cm}^{-1} \text { and } 1050-\right. \\
\left.1260 \mathrm{~cm}^{-1}\right)\end{array}$ & $\sqrt{ }$ & $\sqrt{ }$ & $\sqrt{ }$ & $\sqrt{ }$ & $\sqrt{ }$ & $\sqrt{ }$ \\
\hline bound water $\left(1635-1640 \mathrm{~cm}^{-1}\right)$ & $\sqrt{ }$ & $\sqrt{ }$ & $\sqrt{ }$ & $\sqrt{ }$ & $\sqrt{ }$ & $\sqrt{ }$ \\
\hline $\mathrm{C}-\mathrm{H}$ bending vibration $\left(1455-1460 \mathrm{~cm}^{-1}\right)$ & $\sqrt{ }$ & $\sqrt{ }$ & $\sqrt{ }$ & $x$ & $x$ & $\sqrt{ }$ \\
\hline $\mathrm{C}=0$ summetric stretching vibration $\left(1335-1340 \mathrm{~cm}^{-1}\right)$ & $\sqrt{ }$ & $\sqrt{ }$ & $\sqrt{ }$ & $\sqrt{ }$ & $\sqrt{ }$ & $\sqrt{ }$ \\
\hline C-0 stretching vibration $\left(1070-1450 \mathrm{~cm}^{-1}\right)$ & $\sqrt{ }$ & $\sqrt{ }$ & $\sqrt{ }$ & $\sqrt{ }$ & $\sqrt{ }$ & $\sqrt{ }$ \\
\hline existence of pyranose sugar $\left(910-920 \mathrm{~cm}^{-1}\right)$ & $\sqrt{ }$ & $\sqrt{ }$ & $\sqrt{ }$ & $\sqrt{ }$ & $\sqrt{ }$ & $\sqrt{ }$ \\
\hline $\begin{array}{l}\beta \text {-configuration of pyranose sugar units }\left(864-900 \mathrm{~cm}^{-}\right. \\
1 \text { ) }\end{array}$ & $\sqrt{ }$ & $\sqrt{ }$ & $\sqrt{ }$ & $\sqrt{ }$ & $\sqrt{ }$ & $\sqrt{ }$ \\
\hline
\end{tabular}

The absorption peak at $2920-2935 \mathrm{~cm}^{-1}$ indicated the $\mathrm{C}-\mathrm{H}$ stretching viberation in the sugar ring[24-26]. The absorption peak at approximately $1500-1740 \mathrm{~cm}^{-1}$ and $1050-1260 \mathrm{~cm}^{-1}$ were artributed to $\mathrm{C}=0$ stretching viberation and $\mathrm{O}-\mathrm{H}$ bending vibration, demonstrated the existence of uronic acid[27, 28], corresponded to the assay of uronic acid according to 3.1. The absorption peak of $1635-1640 \mathrm{~cm}^{-1}$ was due to the bound water[29]. The band at $1335-1340 \mathrm{~cm}^{-1}, 1070-1450 \mathrm{~cm}^{-1}$ might be respectively artributed to $C=0$ summetric stretching vibration and $C-0$ stretching vibration. Peaks at $910-920 \mathrm{~cm}^{-1}$ and $864-900$ $\mathrm{cm}^{-1}$ indicated the existence of pyranose sugar[29] and $\beta$-type glycosidic bond[20, 30, 31].

However, C-H bending vibration (1455-1460 $\mathrm{cm}^{-1}$ ) could be observed clearly in ZBP, ZJP, ZTP and ZMP but none in ZHP and ZYP by IR spectrum. This distinct showed no significant diffference which was not enough to trace the 6 jujube species. 


\section{Conclusions}

Polysaccharide, as the unique, rich content and abundant bioactive factors in jujube fruit, attracted a large mount of studies. In previous study, the polysaccharides from different jujube species share dissimilar characterization and bioactivities[3]. Even the genetic method has been significantly efficient in the field of jujube species identification, polysaccharide characterization method also possesses practical significance like recognizing certain jujube species in the mixture. Therefore we aimed to find out an efficient way to trace the botanical origin of jujube species by some significant indicators with crude polysaccharide.

This study demonstrated that the $6 \mathrm{Gl}$ jujube species shared similar monosaccharide composition, functional groups and bonds. In the data of monosaccharide composition, the existence of rhamanose and the abundance of galacturonic acid inferred that pectin might be the main component for the crude polysaccharides from $6 \mathrm{GI}$ jujube species[16, 32]. The FT-IR spectrum analysis revealed that the existence of bond waters and this might explain the existence of $M_{w}$ 240-250 Da, which located between free monosaccharides (150-180 Da) and disaccharides (282-342 Da).

However, the $M_{w}$ distribution exhibited both homogeneity and significant differences among the $6 \mathrm{GI}$ jujube species. In HPGPC profiles, five peaks (RT1, RT2, RT3, RT4, RT5) were almost observed in 6 jujube species and the content of RT3 $(240-250 \mathrm{Da})$, with positive or negative correlation to the content of RT4 and RT5, showed significant differences by RSD and correlation analysis.

In summary, the crude polysaccharides from $6 \mathrm{Gl}$ jujube species of Shanxi province showed homogeneity and significant differences in $\mathrm{M}_{\mathrm{W}}$ distribution but few differences in purity, uronic acid content, monosaccharide composition, and functional groups and bonds. Therefore, the $\mathrm{M}_{\mathrm{w}}$ distribution may be a potential tracing indicator among 6 jujube species.

In order to explore whether this method can be widely used in tracing most jujube species, two deep essential directions should be concerned about in the future researches. First, we should set up continuously updated and opening database of $\mathrm{M}_{\mathrm{w}}$ distribution in different jujube species not just the six jujube fruit mentioned in this article. Second, the same jujube species with different geographical locations and climatic conditions treated should be tested to prove the stability of the $M_{w}$ distribution.

\section{Abbreviations}

$\mathrm{GI}$

Geographical indication

TCM

Traditional Chinese medicine

$\mathrm{M}_{\mathrm{w}}$

Molecular weight 
HPGPC

High-performance gel permeation chromatography

IC

Ion chromatography

NMR

Nuclear magnetic resonance

HPLC-RID

High performance liquid chromatography

GC-MS

Gas chromatography mass spectrometry

FT-IR

Fourier-transform infrared

RSD

Relative standard deviation

ZBP, ZJP, ZTP, ZHP, ZYP and ZMP

Crude polysaccharide of Ziziphus Jujuba cv. Banzao, Ziziphus Jujuba cv. Junzao, Ziziphus Jujuba cv.

Tuntunzao, Ziziphus Jujuba cv. Hupingzao, Ziziphus Jujuba cv. Youzao, and Ziziphus Jujuba cv. Muzao RT1-5

Retention time 1-5.

\section{Declarations}

Ethics approval and consent to participate

Not applicable.

\section{Consent for publication}

Not applicable.

\section{Availability of data and materials}

The datasets used and/or analyzed during the current study are available from the corresponding author on reasonable request.

\section{Competing interests}

The authors declare that they have no competing interests.

\section{Fundings}

This study was supported by Agricultural Research Innovation team Cultivation project of Shanxi Academy of Agricultural Sciences (No. YGC2019TD06), Key R\&D project of Shanxi Province (No. 
201903D211007) and the earmarked fund for Modern Agro-industry Technology Research System.

\section{Authors' contributions}

MK, YC have made substantial contributions to the conception and design of the work; DW, ZJ have collected jujube samples; $H J, Y Z$ have analysed and interpreted $M_{w}$ data; $M K$ has drafted the work; YC, ZL have revised the draft. All authors have read and approved the final manuscript.

\section{Acknowledgements}

We are grateful to the team of fruits \& vegetables research department and the platform of Shanxi Institute for Functional Food, Shanxi Agricultural University.

\section{References}

1. Huang YL, Yen GC, Sheu F, Chau C. Effects of water-soluble carbohydrate concentrate from Chinese jujube on different intestinal and fecal indices. Journal of Agricultural and Food Chemistry. 2008;56: 1734.

2. Soni H, Malik JK: Phyto-Pharmacological Potential of Zizyphus jujube. A Review. Sch Int J Biochem. 2021;4: 1-5.

3. Li JW, Fan LP, Ding SD, Ding XL. Nutritional composition of five cultivars of Chinese jujube. Food chemistry. 2007;103: $454-60$.

4. Gao QH, Wu PT, Liu JR, Wu CS, Parry JW, Wang M. Physico-chemical properties and antioxidant capacity of different jujube (Ziziphus jujuba Mill.) cultivars grown in loess plateau of China. Scientia Horticulturae. 2011;130: 67-72.

5. Pawlowska AM, Camangi F, Bader A, Braca A. Flavonoids of Zizyphus jujuba L. and Zizyphus spinachristi (L.) Willd (Rhamnaceae) fruits. Food Chemistry. 2009;112: 858-62.

6. Guo S, Duan J, Tang Y, Su S, Shang E, Ni S, Qian D. High-performance liquid chromatography-Two wavelength detection of triterpenoid acids from the fruits of Ziziphus jujuba containing various cultivars in different regions and classification using chemometric analysis. Journal of Pharmaceutical and Biomedical Analysis. 2009;49: 1296-302.

7. Guo S, Duan JA, Tang YP, Yang NY, Qian DW, Su SL, Shang EX. Characterization of triterpenic acids in fruits of Ziziphus species by HPLC-ELSD-MS. Journal of agricultural and food chemistry. 2010;58: 6285-9.

8. Gao QH, Wu CS, Wang M. The jujube (Ziziphus jujuba Mill.) fruit: a review of current knowledge of fruit composition and health benefits. Journal of Agricultural and Food Chemistry. 2013;61: 335163.

9. Guo S, Duan JA, Tang Y-P, Zhu ZH, Qian YF, Yang NY, Shang EX, Qian DW. Characterization of nucleosides and nucleobases in fruits of Ziziphus jujuba by UPLC-DAD-MS. Journal of agricultural and food chemistry. 2010;58: 10774-80. 
10. Xu C, Gao J, Du Z, Li D, Wang Z, Li Y, Pang X. Identifying the genetic diversity, genetic structure and a core collection of Ziziphus jujuba Mill. var. jujuba accessions using microsatellite markers. Scientific reports. 2016;6: 1-11.

11. Mazzei P, Piccolo A, Brescia M, Caprio E. Assessment of geographical origin and production period of royal jelly by NMR metabolomics. Chemical and Biological Technologies in Agriculture. 2020;7: 24.

12. Haytowitz D, Lemar L, Pehrsson P, Exler J, Patterson K, Thomas R, Nickle M, Williams J, Showell B, Khan M. USDA national nutrient database for standard reference, release 24. US Department of Agriculture: Washington, DC, USA. 2011.

13. Hou C, Yin M, Lan P, Wang H, Nie H, Ji X. Recent progress in the research of Angelica sinensis (Oliv.) Diels polysaccharides: extraction, purification, structure and bioactivities. Chemical and biological technologies in agriculture. 2021;8: 1-14.

14. Chen L, Huang G. The antiviral activity of polysaccharides and their derivatives. International Journal of Biological Macromolecules. 2018;115: 77-82.

15. Dubois M, Gilles KA, Hamilton JK, Rebers Pt, Smith F. Colorimetric method for determination of sugars and related substances. Analytical chemistry. 1956;28: 350-6.

16. Zhao P, Li X, Wang Y, Yan L, Guo L, Huang L, Gao W. Characterisation and saccharide mapping of polysaccharides from four common Polygonatum spp. Carbohydrate Polymers. 2020;233: 115836.

17. Blumenkrantz N, Asboe-Hansen G. New method for quantitative determination of uronic acids. Analytical biochemistry. 1973;54: 484-9.

18. Wang X, Zhou X, Wang K, Cao X. Structural characterisation and bioactivity of polysaccharides isolated from fermented Dendrobium officinale. Journal of the Science of Food and Agriculture. 2022;102: 280-90.

19. Hu Dj, Cheong Kl, Zhao J, Li Sp. Chromatography in characterization of polysaccharides from medicinal plants and fungi. Journal of Separation Science. 2013;36: 1-19.

20. Li N, Shi C, Shi S, Wang H, Yan J, Wang S. An inulin-type fructan isolated from Artemisia japonica and its anti-arthritic effects. Journal of Functional Foods. 2017;29: 29-36.

21. Guo T, Yang Y, Gao M, Qu Y, Guo X, Liu Y, Cui X, Wang C. Lepidium meyenii Walpers polysaccharide and its cationic derivative re-educate tumor-associated macrophages for synergistic tumor immunotherapy. Carbohydrate Polymers. 2020;250: 116904.

22. Ji X, Cheng Y, Tian J, Zhang S, Jing Y, Shi M. Structural characterization of polysaccharide from jujube (Ziziphus jujuba Mill.) fruit. Chemical and Biological Technologies in Agriculture. 2021;8: 1-7.

23. Ji X, Hou C, Yan Y, Shi M, Liu Y. Comparison of structural characterization and antioxidant activity of polysaccharides from jujube (Ziziphus jujuba Mill.) fruit. International Journal of Biological Macromolecules. 2020;149: 1008-18.

24. Liu XC, Zhu ZY, Tang YL, Wang M-f, Wang Z, Liu AJ, Zhang YM. Structural properties of polysaccharides from cultivated fruit bodies and mycelium of Cordyceps militaris. Carbohydrate Polymers. 2016;142: 63-72. 
25. Wang J, Bao A, Meng X, Guo H, Zhang Y, Zhao Y, Kong W, Liang J, Yao J, Zhang J. An efficient approach to prepare sulfated polysaccharide and evaluation of anti-tumor activities in vitro. Carbohydrate polymers. 2018;184: 366-75.

26. Chen W, Zhu X, Ma J, Zhang M, Wu H. Structural elucidation of a novel pectin-polysaccharide from the petal of Saussurea laniceps and the mechanism of its anti-HBV activity. Carbohydrate polymers. 2019;223: 115077.

27. Chen Y, Xie MY, Nie SP, Li C, Wang YX. Purification, composition analysis and antioxidant activity of a polysaccharide from the fruiting bodies of Ganoderma atrum. Food Chemistry. 2008;107: 231-41.

28. Ji X, Zhang F, Zhang R, Liu F, Peng Q, Wang M. An acidic polysaccharide from Ziziphus Jujuba cv. Muzao: Purification and structural characterization. Food chemistry. 2019;274: 494-9.

29. Zhao G, Kan J, Li Z, Chen Z. Structural features and immunological activity of a polysaccharide from Dioscorea opposita Thunb roots. Carbohydrate Polymers. 2005;61: 125-31.

30. Xing X, Cui SW, Nie S, Phillips GO, Goff HD, Wang Q. Study on Dendrobium officinale O-acetylglucomannan (Dendronan ${ }^{\circledR}$ ): Part I. Extraction, purification, and partial structural characterization. Bioactive Carbohydrates and Dietary Fibre. 2014;4: 74-83.

31. Coimbra MA, Goncalves F, Barros AS, Delgadillo I. Fourier transform infrared spectroscopy and chemometric analysis of white wine polysaccharide extracts. Journal of Agricultural and Food Chemistry. 2002;50: 3405-11.

32. Wang Y, Ding S, Chen F, Xiao G, Fu X, Wang R. Changes in pectin characteristics of jujube fruits cv" Dongzao" and" Jinsixiaozao" during cold storage. Journal of Food Science. 2021;86: 3001-13.

\section{Figures}

\section{Figure 1}

The photos of dried fruits of (a) Ziziphus Jujuba cv. Banzao, (b) Ziziphus Jujuba cv. Junzao, (c) Ziziphus Jujuba cv. Tuntunzao, (d) Ziziphus Jujuba cv. Hupingzao, (e) Ziziphus Jujuba cv. Youzao, (f) Ziziphus Jujuba cv. Muzao.

\section{Figure 2}

The HPGPC profiles of ZBP, ZJP, ZTP, ZHP, ZYP and ZMP. RT1-RT 5 represented 5 peaks in HPGPC profiles, and the peak at about 46 min was solvent peak of $\mathrm{NaCl}$. 


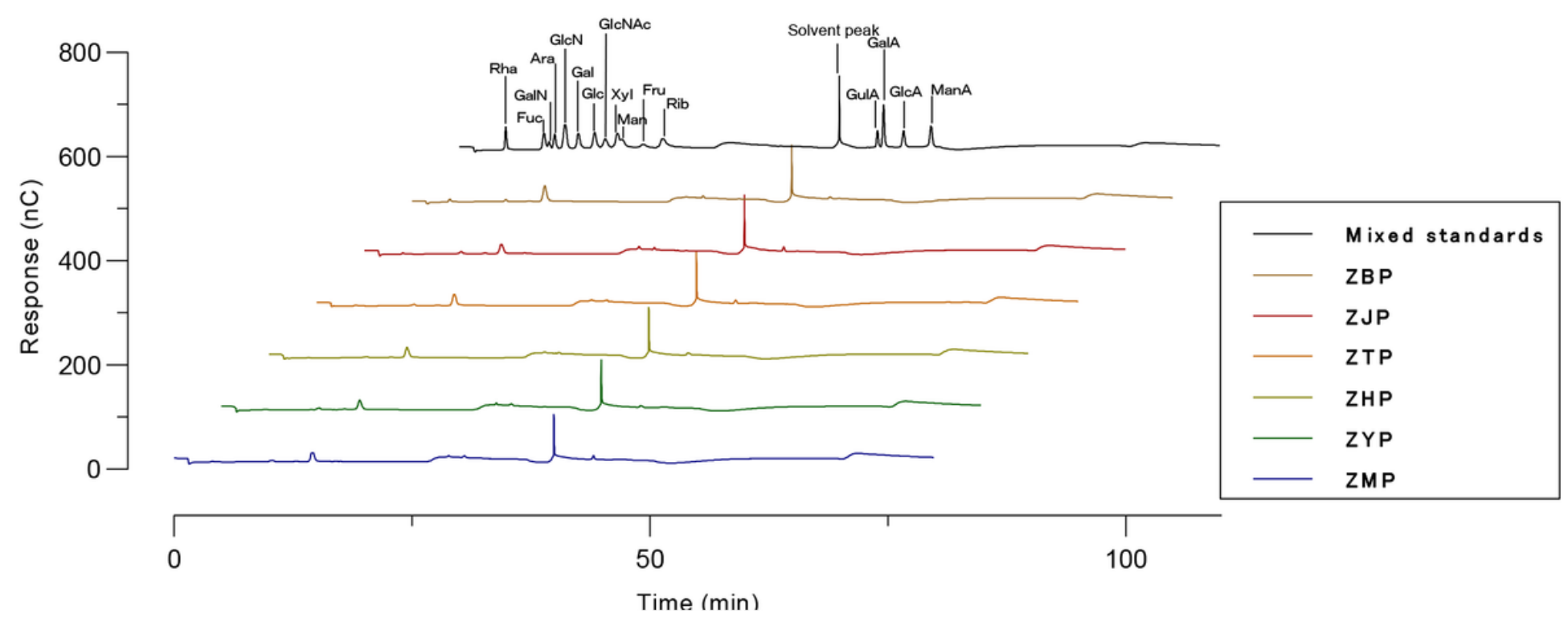

Figure 3

Ion chromatograms profiles of mixed monosaccharides and the crude polysacharide of ZBP, ZJP, ZTP, ZHP, ZYP and ZMP. Black line: 16 monosaccharides standard in mixture were fucose (Fuc), galactosamine (GalN), rhamonose (Rha), arabinose (Ara), glucosamine hydrochloride (GlcN), galactose (Gal), glucose (Glc), N-acetyl-D-glucosamine (GlcNAc), xylose (Xyl), mannose (Man), frutose (Fru), ribose (Rib), galactose acid (GalA), glucuronic acid (GulA), glucose acid (GlcA), mannose acid (ManA) .

\section{Figure 4}

FT-IR spectra of crude polysaccharide in six GI jujube species. The red line: ZBP, the black line: ZJP, the olive green line: ZTP, the green line: ZHP, the blue line: ZYP and the purple line: ZMP.

\section{Supplementary Files}

This is a list of supplementary files associated with this preprint. Click to download.

- Graphicalabstract.png 